\title{
Estructura Factorial del Sentido de Coherencia y su Relación de Apego
}

\author{
Dorys Ortiz-Granja (D), Liliana Jayo-Suquillo(D) \\ Facultad de Psicologa - Pontificia Universidad Catlica del Ecuador, Quito-Pi, Ecuador \\ Carlos Ramos-Galarza ${ }^{1}$ D \\ Facultad de Psicologa - Pontificia Universidad Catlica del Ecuador, Quito-PI, Ecuador \\ Centro de Investigación MIST - Universidad Tecnológica Indoamérica, Quito-Pi, Ecuador
}

\section{RESUMEN}

El sentido de coherencia se conforma por tres factores: comprensibilidad, manejabilidad y significatividad. En la investigación se analizó su estructura factorial a partir de la escala SOC-29 y su relación con de apego. Se plantearon tres hipótesis para valorar la confiabilidad, validez convergente y estructura factorial. La muestra estuvo compuesta por 445 participantes. El análisis estadístico se basó en técnicas descriptivas, alfa de Cronbach, correlación de Pearson y análisis factorial confirmatorio. Los resultados obtenidos aportan evidencias en favor de las hipótesis, con una consistencia interna de $\alpha=.82$, una correlación significativa de apego $r=.27$ a .83 y un ajuste aceptable del modelo $\chi^{2}=1275.88, \mathrm{CFI}=.85, \mathrm{RMSEA}=.07$ [.06-.07], SRMR $=.04$. Terminado el estudio, se ha destacado la importancia del cambio lingüístico realizado para adaptar la escala al contexto ecuatoriano; se mencionan dos limitaciones importantes referentes a su aplicación como autorrelato y la localización geográfica, a considerar para trabajos posteriores.

Palabras clave: Apego; escala SOC; manejabilidad; sentido de coherencia; significado de la vida.

\section{RESUMO - Estrutura Fatorial do Senso de Coerência e sua Relação com o Apego}

O senso de coerência é formado por três fatores: compreensibilidade, gerenciabilidade e significância. Na pesquisa, sua estrutura fatorial foi analisada por ser avaliada com a escala SOC-29 e sua relação com o apego. Três hipóteses foram propostas para avaliar confiabilidade, validade convergente e estrutura fatorial. A amostra foi composta por 445 participantes. A análise estatística foi baseada em técnicas descritivas, alfa de Cronbach, correlação de Pearson e análise fatorial confirmatória. Os resultados fornecem evidência para as hipóteses com uma consistência interna de $\alpha=0,82$, uma correlação significativa $r=0,27$ a 0,83 e ajuste aceitável do modelo $\chi^{2}=1275.88, \mathrm{CFI}=0,85, \mathrm{RMSEA}=0,07$ [0,06-0,07], SRMR =0,04. O artigo está terminado, enfatizando a importância da mudança linguística feita para adaptar a escala ao contexto equatoriano. Duas limitações importantes são mencionadas em relação à sua aplicação como autorrelato e localização geográfica, a serem levadas em conta para o trabalho subsequente.

Palavras-chaves: apego; escala SOC; gerenciabilidade; senso de coerência; significado da vida.

\section{ABSTRACT - Factorial Structure of the Sense of Coherence and its Relation with Attachment}

The Sense of Coherence is formed by three factors: comprehensibility, manageability and meaningfulness. In this study, its factorial structure was analyzed through assessment with the SOC-29 scale and its relationship with attachment. Three hypotheses were proposed to assess the reliability, convergent validity and factorial structure, using a sample of 445 participants. Statistical analysis was based on descriptive techniques, Cronbach's alpha, Pearson's correlation and confirmatory factor analysis. The results obtained provide evidence in favor of the hypotheses, with an internal consistency of $\alpha=.82$, a significant correlation with attachment $r=.27$ to .83 and an acceptable fit of the model $\chi^{2}=1275.88, \mathrm{CFI}=.85, \mathrm{RMSEA}=.07$ [.06-.07], SRMR $=.04$. The study highlights the importance of the linguistic changes made to adapt the scale to the Ecuadorian context. Two important limitations are mentioned regarding its application as a self-report and the geographical location, which need to be taken into consideration in subsequent studies.

Keywords: Attachment; manageability; meaning of life; SOC scale; sense of coherence.

Durante muchos años, la psicología se ha interesado por la enfermedad y centrado sus reflexiones, estudios e investigaciones en los problemas y en las dificultades de los seres humanos (Gómez, 2017). A partir de los años 50 surge un movimiento interesado en los aspectos positivos y creativos del ser humano que potencian su bienestar y desarrollo que se ha denominado como psicología positiva (Seligman, 1991).
Así, comienzan a plantearse una serie de inquietudes acerca de la salud y de los factores implicados en ella. En las últimas décadas del siglo XX aparece el término salutogénesis planteado por Antonovsky (1987) en el que se combina los términos "salud" y "génesis". Este enfoque se centra en los factores humanos que apoyan la salud y el bienestar que permiten a los seres humanos enfrentar el estrés y los problemas de forma positiva (Gómez, 2017). 
Los estudios realizados en base a esta teoría (Salazar, Ruvalcaba, Figueroa, \& Pando, 2015; Ureña, 2008) contribuyen a determinar que existen personas que enfrentan mejor que otras, las tensiones de la vida diaria. Antonovsky (1987) denominó a esta capacidad el Sentido de Coherencia, que implica una forma particular de enfrentar la vida y sus desafíos (Ureña \& Castro, 2009).

Según Antonovsky (1996) el Sentido de Coherencia está conformado por tres factores: la comprensibilidad, la manejabilidad y la significatividad. El primero es el componente cognitivo que le permite a la persona ordenar, predecir y explicar los estímulos que recibe. El segundo es un componente instrumental que describe el nivel de convicción que tiene cada persona para superar las situaciones que se le presenten, por sí misma o con la ayuda de otras personas y, finalmente, el tercer factor tiene que ver con el sentido que la experiencia adquiere en la propia vida de la persona.

El constructo Sentido de Coherencia fue operacionalizado por Antonovsky, en 1993, en una escala conocida como SOC-29 y, posteriormente, se ha elaborado una versión reducida denominada SOC-13, la cual también ha reportado propiedades psicométricas adecuadas para su aplicación en universitarios. Por ejemplo, la primera versión fue validada por el mismo autor, en 20 países mediante la realización de 26 estudios, mostrando un Alpha de Cronbach de .82 a .95 , indicando un alto nivel de validez interna (Lizarbe-Chocarro, Guillén-Grima, Aguinaga-Ontoso, \& Canga-Armayor, 2016).

Sin embargo, una validación de la escala SOC-29, en adolescentes españoles (Rivera, López, Ramos, \& Moreno, 2011) no mostró buenos índices de validez y en otro estudio (Flensborg-Madsen, Ventegodt, \& Merrick, 2005) no se encontró una asociación entre el SOC e indicadores de salud física, por lo que se propuso una escala alternativa de 20 ítems y la necesidad de validar nuevas versiones que tengan una mejor validez interna. Posteriormente, se elaboró una escala de 13 ítems (SOC-13), la misma que fue puesta a prueba por Virués, Martínez, Barrio y Lozano (2007), en una población de 419 ancianos españoles, mayores de 70 años, determinando que es válida y confiable para dicho rango de población, incluso con bajos niveles de instrucción. Resultados similares fueron obtenidos por Lizarbe-Chocarro et al. (2016) y Velásquez, Cárdenas, Chávez, y Montes de Oca (2014), quienes han aportado en favor de las adecuadas propiedades psicométricas de la SOC. Además, se ha reportado que el cuestionario SOC en su versión 13 tendría mayor validez al ser aplicado en poblaciones jóvenes y adolescentes; datos que se confirman además, con una validación realizada en una muestra de jóvenes universitarios peruanos entre 18-29 años (Saravia, Ibérico, \& Yearwood, 2014).

Eriksson y Lindström (2005) llevaron a cabo una revisión acerca de la aplicación de la escala SOC indicando que se ha usado en 33 idiomas diferentes. En dicho estudio se reportó que su Alpha de Cronbach alcanza entre .70 a .95 , el re-test también muestra estabilidad con rangos entre .69 y .78 (un año) y hasta .54 (10 años), indicando que el SOC tiende a aumentar conforme avanza la edad. La revisión concluye señalando que la escala tiene características adecuadas de fiabilidad, validez y puede ser aplicable en diferentes culturas.

Si bien, la escala SOC ha sido ampliamente utilizada en el contexto clínico, laboral y educativo, su contenido está formulado desde proposiciones negativas, lo cual, a nuestro criterio podría generar fenómenos como la sugestión negativa, persuasión, complicación en la valoración cuantitativa del aspecto negativo, direccionamiento hacia respuestas poco genuinas, sesgo de protección de la identidad de la persona y por ende puntuar bajo en la escala. En tal sentido, surge una primera necesidad, que consiste en proponer una escala SOC que contenga ítems con una formulación positiva, por lo tanto, este será uno de nuestros fines de investigación.

El hecho de modificar los ítems de la escala a positivo plantea algunos beneficios. A nivel de la escala misma favorece una mayor claridad en los ítems, lo que redundará en que las respuestas estén más acordes con la experiencia de los participantes. Otro beneficio importante es aumentar su nivel de coherencia interna puesto que todos los ítems están formulados en el mismo sentido (positivo) y no existe una diferencia en este; es decir, algunos hacia un extremo y los demás hacia el otro, lo que puede generar confusión. Finalmente, otro beneficio que, por estar al último no es menos despreciable, es que la información se procesa mucho más fácilmente a nivel cognitivo (Watzlawick, 2012).

A continuación, presentamos estudios sobre el Sentido de Coherencia y su asociación con otras variables de interés en esta línea de investigación. En trabajos previos realizados por Besteiro et al. (2008) y Hernández, Ehrenzweig, y Yépez (2010) encontraron que los rasgos de la personalidad, el sentido de coherencia y la salud percibida en pacientes con afectación de la salud, existen características que favorecen la enfermedad y distorsionan su percepción, como: alto neuroticismo, débil sentido de coherencia y niveles bajos en la comprensibilidad y el significado. Dematteis, Grill y Posada (2012) determinaron que los adultos en riesgo psicosocial con mayor sentido de coherencia, perciben lo adverso como un desafío, tienen mayor flexibilidad y estabilidad y ante el estrés, reaccionan con calma, poseen alta tolerancia ante la frustración, un sentido de competencia, autoestima, motivación, persistencia, control y organización.

En investigaciones que han relacionado el sentido de coherencia y satisfacción laboral (Malagón, 2016; Mantas, 2017; Ureña, 2009) se ha identificado que a mayor grado de satisfacción laboral, hay un alto optimismo del futuro, satisfacción con la vida personal, mayor actitud positiva de ayuda a otros, más resiliencia, seguridad 
personal y capacidad de adaptación a los cambios y relaciones interpersonales. Es decir, existe una correlación positiva entre las variables sentido de coherencia, resiliencia y salud mental positiva, que permiten concluir que comparten aspectos comunes.

En investigaciones que han analizado el sentido de coherencia de cuidadores de adultos mayores (Dejo, 2007; López-Martínez, Frías-Osuna, \& Del PinoCasado, 2017), se ha encontrado que, el mayor sentido de coherencia genera (a) menor posibilidad de percepción de sobrecarga, (b) tendencia de redefinir la situación, (c) representarla como aceptable y (d) menor probabilidad de conductas desadaptativas y nocivas.

Finalmente, en estudios donde se ha investigado el sentido de coherencia en población juvenil, se ha encontrado que, los jóvenes con altos niveles de SOC identifican como activo de salud el deporte, actitud positiva, la familia y los amigos (Paredes-Carbonell, Agulló-Cantos, Vera-Remartínez, \& Hernán-García, 2013), mientras que, en universitarios se ha reportado que el sentido de coherencia es una fuente que reduce el estrés, promociona la vida saludable y configura niveles bajos de cansancio emocional, sobre todo en varones versus mujeres y en estudiantes de cursos superiores (Martínez, Liébana, \& Morán, 2017).

Como se puede observar, tanto en los estudios de validación de la escala, como aquellos llevados a cabo en diferentes problemáticas, se trata de una línea de investigación no concluyente, puesto que el Sentido de Coherencia se asocia con muchos aspectos como los descritos previamente, siendo uno de los vacíos de esta línea de investigación, el no contar con instrumentos validados en el contexto latinoamericano y específicamente en Ecuador. Los aportes que esta investigación realiza en el contexto local, tiene que ver con: (a) contar con un instrumento validado para evaluar el sentido de coherencia, (b) su uso libre por parte de otros investigadores de Ecuador y otros contextos de habla hispana, (c) la posibilidad de que la línea de investigación sobre el sentido de coherencia siga creciendo en el contexto Latinoamericano, al tener un instrumento accesible para la realización de nuevos estudios y (d) la oportunidad que estudiantes de programas de pre y postgrado de Ecuador puedan aplicar este instrumento como operacionalización de la variable sentido de coherencia, dentro de sus trabajos de titulación. Por tanto, resulta necesario ejecutar esta investigación que tiene como finalidad validar la escala de sentido de coherencia - SOC 29 en el contexto ecuatoriano.

Es importante resaltar ciertas características de Ecuador que hacen que los resultados de esta investigación no sean exclusivos o aislados únicamente para el contexto de este país, como por ejemplo, Ecuador maneja un sistema económico capitalista con el dólar americano como moneda oficial, la religión con mayor prevalencia en el país es la católica romana y su sistema educativo superior maneja un sistema similar al de otros países de la región, por tal razón, los resultados que se presentan a continuación, podrían servir de base para comprender el sentido de coherencia en otros países que compartan características sociales similares.

\section{Hipótesis de Investigación}

$\mathrm{H}_{1}$ La escala SOC modificada y adaptada lingüísticamente al contexto ecuatoriano, presentará valores adecuados de confiabilidad, al ser evaluada mediante el procedimiento de consistencia interna.

$\mathrm{H}_{2}$ La escala SOC tendrá un adecuado valor de validez convergente al ser correlacionada con una escala que evalúe un constructo similar, como lo es una escala para valorar el apego.

$\mathrm{H}_{3}$ La escala SOC en su versión modificada y adaptada al contexto lingüístico de Ecuador presentará valores de bondad de ajuste adecuados en su estructura factorial de 3 dimensiones: significado, comprensión y manejo.

\section{Método}

\section{Participantes}

En un principio se contó con 449 participantes, sin embargo, 4 personas no completaron el consentimiento informado de participación voluntaria, por lo cual, se trabajó con las respuestas de 445 participantes. En cuanto al género participaron 145 hombres (32.5\%) y 300 mujeres (67.4\%). En cuanto a edad, 187 (42\%) estuvieron entre 15 y 20 años de edad, 14 (31.9\%) estuvieron entre 21 y 25 años de edad, 38 (8.5\%) entre 26 y 30 años de edad, 10 (2.2\%) entre 31 y 35 años de edad, 20 (4.5\%) entre 36 y 40 años de edad, 14 (3.1\%) entre 41 y 45 años de edad y $34(7.6 \%)$ más de 46 años de edad. En cuanto a estado civil, $357(80.2 \%)$ participantes eran solteros, 58 (13\%) casado, 14 (3.1\%) divorciados y 16 (3.8\%) unión libre. En cuanto a nivel educativo 67 (15.5\%) contaban con un nivel secundario, 317 (71.2\%) un nivel universitario y 61 (13.7\%) un nivel de postgrado.

\section{Instrumentos}

La Escala SOC-29 es un instrumento que evalúa el Sentido de Coherencia. Está conformada por 29 ítems, distribuidos en tres sub-escalas que sirven para evaluar los constructos que conforman el Sentido de Coherencia: comprensión, manejabilidad y significado; es una escala de uso libre.

Además, se usó una escala de Apego (Ortiz-Granja, 2016) que consta de 12 ítems, referentes a los tres tipos de apego: seguro, evitativo y ambivalente basado en la teoría de Bowlby (1998). En la tabla 2 se presenta la escala de Apego aplicada. 
Tabla 1

Escala SOC

\begin{tabular}{|c|c|c|c|}
\hline Escala & Factor & $\begin{array}{c}\text { No. } \\
\text { Ítem }\end{array}$ & Pregunta \\
\hline \multirow{29}{*}{ SOC } & \multirow{10}{*}{ 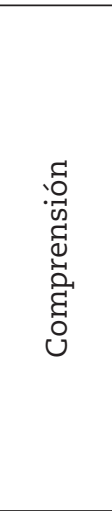 } & 1 & Cuando hablas con los demás ¿sientes que te comprenden? \\
\hline & & 2 & $\begin{array}{l}\text { Cuanto te has encontrado en una situación en la que tenías que hacer algo que } \\
\text { dependía de la colaboración de los demás, has pensado que }\end{array}$ \\
\hline & & 3 & Cómo crees que conocer a la mayor parte de las personas \\
\hline & & 4 & ¿Tienes la sensación de que es importante lo que sucede a tu alrededor? \\
\hline & & 5 & ¿Consideras que puedes confiar en el comportamiento de las personas que conoces bien? \\
\hline & & 8 & Hasta la fecha, tu vida ha estado \\
\hline & & 18 & Cuando te ha sucedido algo satisfactorio sueles \\
\hline & & 19 & Tienes claridad en tus ideas y sentimientos \\
\hline & & 23 & Crees que vas a tener personas con las que contar en el futuro \\
\hline & & 25 & ¿Con qué frecuencia has sentido que eres importante para la gente a tu alrededor? \\
\hline & \multirow{10}{*}{$\begin{array}{l}\stackrel{\circ}{\mathbb{\Xi}} \\
\stackrel{\mathbb{\varpi}}{\Sigma}\end{array}$} & 6 & ¿Ha ocurrido que las personas con las que contabas te han apoyado? \\
\hline & & 10 & En los últimos años tu vida ha estado \\
\hline & & 11 & La mayor parte de las cosas que hagas en el futuro van a ser? \\
\hline & & 12 & ¿Actualmente, te encuentras en una situación en la que sabes lo que tienes que hacer? \\
\hline & & 16 & Realizar las actividades de cada día es \\
\hline & & 17 & Tu vida en el futuro puede estar \\
\hline & & 21 & Sientes que puedes manejar adecuadamente tus sentimientos \\
\hline & & 24 & ¿Tienes la sensación de saber lo que sucederá en el futuro? \\
\hline & & 27 & Cuando piensas en afrontar las dificultades de la vida, tienes la sensación de que \\
\hline & & 29 & ¿Con qué frecuencia tienes la sensación de poder mantener el control? \\
\hline & \multirow{9}{*}{ 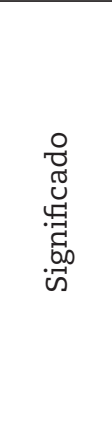 } & 7 & La vida es \\
\hline & & 9 & Crees que se te trata de forma justa \\
\hline & & 13 & ¿De qué forma se describe mejor tu opinión sobre la vida? \\
\hline & & 14 & Cuando piensas acerca de la vida, a menudo \\
\hline & & 15 & Cuando te enfrentas a un problema difícil, encontrar una solución es \\
\hline & & 20 & Cuando haces algo que te produce una buena sensación \\
\hline & & 22 & Esperas que tu vida personal en el futuro sea \\
\hline & & 26 & Cuando algo sucede has notado que \\
\hline & & 28 & Con qué frecuencia tienes la sensación de que las cosas que haces en tu vida tienen sentido \\
\hline
\end{tabular}

Tabla 2

Escala de Valoración del Apego

\begin{tabular}{lcl}
\hline \multicolumn{1}{c}{ Factor } & Ítem & Pregunta \\
\hline \multirow{2}{*}{ Seguro } & 30 & Me es fácil comprometerme emocionalmente con mi pareja \\
& 39 & Me siento mal cuando tengo relaciones afectivas/románticas pasajeras \\
& 41 & Me preocupa poco sentirme rechazado/a por mi pareja \\
Evitativo & 31 & Aunque quiera, es difícil para mí confiar en mi pareja \\
& 32 & Deseo relacionarme románticamente con alguien, pero en general me siento rechazado \\
& 33 & Me siento bien cuando evito compromisos emocionales con otra persona \\
& 36 & Necesito sentirme independiente, sin compromisos afectivos \\
& 40 & Me incomoda depender emocionalmente de mi pareja \\
& 34 & Pienso que las relaciones afectivas pueden lastimar \\
Ambivalente & 35 & Siento que mis parejas no me valoran como yo las valoro a ellas \\
& 37 & Prefiero estar solo/a a tener una relación sentimental estable \\
& 38 & Comprometerme en relaciones afectivas/románticas me da miedo \\
\hline
\end{tabular}




\section{Procedimiento}

Para llevar a cabo la investigación, se realizó en primer lugar un análisis lingüístico de las preguntas de la escala original y se la adaptó tanto semántica como sintácticamente, para alcanzar una mejor comprensión de cada pregunta. Luego se elaboró el cuestionario en el formato de una plataforma tecnológica y se envió a los participantes. Una vez completados los instrumentos, se procedió a realizar su análisis estadístico.

\section{Análisis de Datos}

Para analizar los datos obtenidos se utilizaron las técnicas estadísticas descriptivas de tendencia central y dispersión. Para procesar la consistencia interna de la escala, utilizamos el proceso de Alfa de Cronbach, correlación entre los ítems y escala a la que pertenecen y mejora del coeficiente de consistencia interna si se elimina el ítem. Para evaluar la validez convergente se utilizó el procedimiento de correlación de Pearson para valorar la relación entre las dimensiones de las dos escalas aplicadas. Estos análisis se los realizó en el paquete estadístico
SPSS versión 25. Para el análisis factorial confirmatorio se utilizó el proceso de máxima verosimilitud, en base a los indicadores $\chi^{2}$, CFI, RMSEA y SRMR. El valor de significación estadística fue menor a .01 para la comprobación de la hipótesis planteada.

\section{Resultados}

\section{Propuesta Lingüística de la escala SOC}

El análisis lingüístico de la escala SOC fue realizado en torno a tres parámetros: (a) hay preguntas que no fueron modificadas y se mantuvieron de igual forma que en el instrumento original (Rivera et al., 2011), tales como: $7,8,10,11,13,14,15,16,17,20$ y 22 ; (b) otras preguntas que estaban formuladas en negativo se reformularon en positivo (ver tabla 3), debido a que es más fácil, desde el punto de vista cognitivo, que el cerebro procese información positiva (Watzlawick, 2012) y (c) existen otros ítems que fueron modificados para simplificar su formulación, ya sea porque era muy larga o complicada de entender (ver tabla 4).

Tabla 3

Propuesta de Modificación Lingüística en Formulación Positiva de la Escala SOC

\begin{tabular}{cll}
\hline Ítem & \multicolumn{1}{c}{ Escala original } & \multicolumn{1}{c}{ Escala modificada } \\
\hline 1 & $\begin{array}{l}\text { ¿Cuando hablas con los demás, } \\
\text { sientes que no te comprenden? }\end{array}$ & $\begin{array}{l}\text { ¿Cuando hablas con los } \\
\text { demás, sientes que te } \\
\text { comprenden? }\end{array}$ \\
& $\begin{array}{l}\text { ¿Tienes la sensación de que en } \\
\text { realidad no te importa lo que } \\
\text { sucede a tu alrededor? }\end{array}$ & $\begin{array}{l}\text { ¿Tienes la sensación de } \\
\text { que es importante lo que } \\
\text { sucede a tu alrededor? }\end{array}$
\end{tabular}

$6 \quad$ ¿Ha ocurrido que las personas con las que contabas te han decepcionado?

$¿$ ¿Ha ocurrido que las personas con las que contabas te han apoyado?

¿Crees que se te trata de forma justa?

¿Actualmente, te encuentras en una situación en la que sabes lo que tienes que hacer? situación poco frecuente y que no sabes qué hacer?

18 Cuando algo poco satisfactorio ha sucedido en el pasado sueles:

19 ¿Tienes muchas ideas y sentimientos que se mezclan?

21 ¿Sucede que tienes sentimientos en tu interior que preferirías no tenerlos?
Cuando te ha sucedido algo satisfactorio sueles

¿Tienes claridad en tus ideas y sentimientos?

¿Sientes que puedes manejar adecuadamente tus sentimientos?
La frase se puso en positivo para facilitar su entendimiento por los participantes. Esto favorece el nivel de comprensión.

La frase en positivo mejora la comprensión de la situación y su manejo. Si una persona percibe las situaciones como "poco importantes" probablemente su reacción es de indiferencia.

Se busca el aspecto positivo en las relaciones, basados en la idea de que el apoyo brinda mejores oportunidades de crecimiento y desarrollo personal.

La frase en positivo mejora la comprensión de la situación.

La frase en positivo mejora las posibilidades de manejo de la situación.

La frase en positivo expresa mejor la capacidad de manejar la situación de una persona.

La claridad en el pensamiento es importante para mejorar el nivel de comprensión de la situación y, por lo tanto, su manejo.

Centrarse en el aspecto positivo, mejora el sentido de autoeficacia de las personas. 
Tabla 3 (continuación)

Propuesta de Modificación Lingüística en Formulación Positiva de la Escala SOC

\begin{tabular}{|c|c|c|c|}
\hline Ítem & Escala original & Escala modificada & Argumento \\
\hline 24 & $\begin{array}{l}\text { ¿Te sucede que tienes la sensación } \\
\text { de que no sabes exactamente qué } \\
\text { va a suceder? }\end{array}$ & $\begin{array}{l}\text { ¿Tienes la sensación de } \\
\text { saber lo que sucederá en } \\
\text { el futuro? }\end{array}$ & $\begin{array}{l}\text { La confianza en el futuro mejora la } \\
\text { capacidad de respuesta de una persona y } \\
\text { apoya el significado que un evento tiene } \\
\text { para ella. }\end{array}$ \\
\hline 25 & $\begin{array}{l}\text { Muchas personas, incluso aquellas } \\
\text { con fuerte carácter, se sienten } \\
\text { algunas veces como si fuesen } \\
\text { un cero a la izquierda en ciertas } \\
\text { situaciones. ¿Con qué frecuencia te } \\
\text { has sentido así en el pasado? }\end{array}$ & $\begin{array}{l}\text { ¿Con qué frecuencia } \\
\text { has sentido que eres } \\
\text { importante para la gente } \\
\text { a tu alrededor? }\end{array}$ & $\begin{array}{l}\text { Se enfatiza el aspecto positivo de las } \\
\text { situaciones para mejorar su manejo y } \\
\text { significado. }\end{array}$ \\
\hline 28 & $\begin{array}{l}\text { ¿Con qué frecuencia tienes la } \\
\text { sensación de que tienen poco } \\
\text { sentido las cosas que haces en tu } \\
\text { vida cotidiana? }\end{array}$ & $\begin{array}{l}\text { ¿Con qué frecuencia } \\
\text { tienes la sensación de } \\
\text { que las cosas que haces } \\
\text { en tu vida tienen sentido? }\end{array}$ & $\begin{array}{l}\text { Lograr que las cosas tengan un propósito } \\
\text { y un significado es importante para } \\
\text { alcanzarlas. }\end{array}$ \\
\hline 29 & $\begin{array}{l}\text { ¿Con qué frecuencia tienes } \\
\text { sensaciones de no estar seguro/a } \\
\text { de poder mantener el control? }\end{array}$ & $\begin{array}{l}\text { ¿Con qué frecuencia } \\
\text { tienes la sensación } \\
\text { de poder mantener el } \\
\text { control? }\end{array}$ & $\begin{array}{l}\text { Es importante que la persona perciba que } \\
\text { puede tener las cosas bajo control para } \\
\text { que pueda manejarlas. }\end{array}$ \\
\hline
\end{tabular}

Tabla 4

Propuesta de Modificación Lingüística en Formulación Simplificada de la Escala SOC

\begin{tabular}{|c|c|c|c|}
\hline Ítem & Escala original & Escala modificada & Observación \\
\hline 2 & $\begin{array}{l}\text { En el pasado, cuando te has encontrado en } \\
\text { una situación en la que tenías que hacer } \\
\text { algo que dependía de la colaboración de } \\
\text { los demás, has pensado que: }\end{array}$ & $\begin{array}{l}\text { Cuanto te has encontrado en una } \\
\text { situación en la que tenías que } \\
\text { hacer algo que dependía de la } \\
\text { colaboración de los demás, has } \\
\text { pensado que }\end{array}$ & Se formuló en presente \\
\hline 3 & $\begin{array}{l}\text { Piensa en las personas con las que estás } \\
\text { en contacto diario, aparte de aquellas a las } \\
\text { que consideras más cercanas. ¿Cómo crees } \\
\text { que conoces a la mayor parte de éstas? }\end{array}$ & $\begin{array}{l}\text { Cómo crees que conocer a la } \\
\text { mayor parte de las personas }\end{array}$ & $\begin{array}{l}\text { Se dejó solo la pregunta, } \\
\text { simplificando su } \\
\text { comprensión }\end{array}$ \\
\hline 5 & $\begin{array}{l}\text { ¿Ha sucedido en el pasado que te haya } \\
\text { sorprendido el comportamiento de las } \\
\text { personas que pensabas conocer bien? }\end{array}$ & $\begin{array}{l}\text { Consideras que puedes confiar } \\
\text { en el comportamiento de las } \\
\text { pesonas que conoces bien? }\end{array}$ & $\begin{array}{l}\text { Se reformuló para facilitar } \\
\text { la comprensión }\end{array}$ \\
\hline 23 & $\begin{array}{l}\text { ¿Crees que siempre vas a tener personas } \\
\text { con las que poder contar en el futuro? }\end{array}$ & $\begin{array}{l}\text { Crees que vas a tener personas } \\
\text { con las que contar en el futuro }\end{array}$ & Se aclaró \\
\hline 26 & $\begin{array}{l}\text { Cuando algo sucede, has comprobado por } \\
\text { lo general que: }\end{array}$ & $\begin{array}{l}\text { Cuando algo sucede has notado } \\
\text { que }\end{array}$ & $\begin{array}{l}\text { Se cambió "comprobación" } \\
\text { por "notado" }\end{array}$ \\
\hline 27 & $\begin{array}{l}\text { Cuando piensas en dificultades } \\
\text { que pudieras afrontar en aspectos } \\
\text { importantes de tu vida, tienes la } \\
\text { sensación de que: }\end{array}$ & $\begin{array}{l}\text { Cuando piensas en afrontar las } \\
\text { dificultades de la vida, tienes la } \\
\text { sensación de que }\end{array}$ & $\begin{array}{l}\text { Se simplificó la frase para } \\
\text { facilitar su comprensión }\end{array}$ \\
\hline
\end{tabular}

\section{Consistencia Interna de cada escala del SOC \\ Escala de Comprensión. Esta escala se conforma} por los ítems 1, 2, 3, 4, 5, 8, 18, 19, 23 y 25. El coeficiente de Alfa de Cronbach de la escala fue $\alpha=.82$. En la tabla 5 se presenta la correlación entre los ítems que conforman la escala y como mejoraría el Alfa si alguno de sus ítems fuera eliminado.

Escala de Manejo. Esta escala se compone de los ítems 6, 10, 11, 12, 16, 17, 21, 24, 27 y 29. El Alfa de Cronbach de la escala de Manejo fue de $\alpha=.86$. En la tabla 6 se presenta la correlación entre los ítems de la escala y su análisis de consistencia interna. 
Tabla 5

Escala de Comprensión

\begin{tabular}{cccccc}
\hline Ítems & $\begin{array}{c}\text { Media de escala si } \\
\text { el elemento se ha } \\
\text { suprimido }\end{array}$ & $\begin{array}{c}\text { Varianza de escala } \\
\text { si el elemento se ha } \\
\text { suprimido }\end{array}$ & $\begin{array}{c}\text { Correlación total de } \\
\text { elementos corregida }\end{array}$ & $\begin{array}{c}\text { Correlación } \\
\text { múltiple al } \\
\text { cuadrado }\end{array}$ & $\begin{array}{c}\text { Alfa de Cronbach si } \\
\text { el elemento se ha } \\
\text { suprimido }\end{array}$ \\
\hline 1 & 34.74 & 27.99 & .57 & .40 & .79 \\
2 & 34.77 & 27.57 & .57 & .37 & .79 \\
3 & 35.09 & 28.60 & .51 & .37 & .80 \\
4 & 34.63 & 28.75 & .28 & .11 & .81 \\
5 & 34.83 & 29.75 & .49 & .29 & .82 \\
8 & 34.45 & 27.92 & .49 & .29 & .80 \\
18 & 34.09 & 28.50 & .52 & .33 & .80 \\
19 & 34.59 & 27.16 & .56 & .43 & .80 \\
23 & 34.21 & 27.25 & .63 & .49 & .79 \\
25 & 34.77 & 25.92 & & 78 \\
\hline
\end{tabular}

Tabla 6

Escala de Manejo

\begin{tabular}{cccccc}
\hline Ítems & $\begin{array}{c}\text { Media de escala si } \\
\text { el elemento se ha } \\
\text { suprimido }\end{array}$ & $\begin{array}{c}\text { Varianza de escala } \\
\text { si el elemento se ha } \\
\text { suprimido }\end{array}$ & $\begin{array}{c}\text { Correlación total de } \\
\text { elementos corregida }\end{array}$ & $\begin{array}{c}\text { Correlación } \\
\text { múltiple al } \\
\text { cuadrado }\end{array}$ & $\begin{array}{c}\text { Alfa de Cronbach si } \\
\text { el elemento se ha } \\
\text { suprimido }\end{array}$ \\
\hline 6 & 33.03 & 38.09 & .37 & .17 & .39 \\
10 & 33.53 & 34.61 & .61 & .49 & .83 \\
11 & 32.71 & 37.83 & .58 & .41 & .89 \\
12 & 33.01 & 34.93 & .69 & .49 & .84 \\
16 & 33.23 & 34.99 & .62 & .46 & .83 \\
17 & 33.47 & 33.83 & .32 & .26 & .83 \\
21 & 33.37 & 34.25 & .64 & .44 & .83 \\
24 & 33.78 & 36.58 & .61 & .43 & .86 \\
27 & 33.04 & 34.70 & 35.32 & & .83 \\
29 & 33.31 & & & & .83 \\
\hline
\end{tabular}

Escala de Significado. Esta escala está conformada por los ítems 7, 9, 13, 14, 15, 20, 22, 26 y 28. El Alfa de Cronbach de la escala fue $\alpha=.85$. En la tabla 7 se presenta la correlación entre los ítems de la escala y su análisis de consistencia interna.

Escala de Apego Seguro. Esta escala está conformada por los ítems 1, 10 y 12. El Alfa de Cronbach de la escala fue $\alpha=.12$ y eliminar alguno de los ítems no mejoraba la escala, ni las correlaciones entre sus ítems fueron adecuadas, por lo cual, se descartó esta variable en el resto de análisis.

Escala de Apego Evitativo. Esta escala se conforma por los ítems 2, 3, 4, 7 y 11. El Alfa de Cronbach de la escala fue $\alpha=.71$. En la tabla 8 se presenta la correlación entre los ítems de la escala y su análisis de consistencia interna.

Tabla 7

Escala de Comprensión

\begin{tabular}{cccccc}
\hline Ítems & $\begin{array}{c}\text { Media de escala si } \\
\text { el elemento se ha } \\
\text { suprimido }\end{array}$ & $\begin{array}{c}\text { Varianza de escala } \\
\text { si el elemento se ha } \\
\text { suprimido }\end{array}$ & $\begin{array}{c}\text { Correlación total de } \\
\text { elementos corregida }\end{array}$ & $\begin{array}{c}\text { Correlación } \\
\text { múltiple al } \\
\text { cuadrado }\end{array}$ & $\begin{array}{c}\text { Alfa de Cronbach si } \\
\text { el elemento se ha } \\
\text { suprimido }\end{array}$ \\
\hline 7 & 30.99 & 27.90 & .60 & .42 & .20 \\
9 & 31.32 & 30.40 & .40 & .41 & .83 \\
13 & 30.79 & 28.81 & .63 & .52 & .83 \\
14 & 31.09 & 26.67 & .55 & .35 & .82 \\
15 & 31.39 & 29.09 & .60 & .38 & .84 \\
20 & 30.88 & 28.36 & & & .83 \\
\hline
\end{tabular}


Tabla 7 (continuación)

Escala de Comprensión

\begin{tabular}{cccccc}
\hline Ítems & $\begin{array}{c}\text { Media de escala si } \\
\text { el elemento se ha } \\
\text { suprimido }\end{array}$ & $\begin{array}{c}\text { Varianza de escala } \\
\text { si el elemento se ha } \\
\text { suprimido }\end{array}$ & $\begin{array}{c}\text { Correlación total de } \\
\text { elementos corregida }\end{array}$ & $\begin{array}{c}\text { Correlación } \\
\text { múltiple al } \\
\text { cuadrado }\end{array}$ & $\begin{array}{c}\text { Alfa de Cronbach si } \\
\text { el elemento se ha } \\
\text { suprimido }\end{array}$ \\
\hline 22. & 30.46 & 30.99 & .42 & .23 & .85 \\
26 & 31.45 & 27.99 & .57 & .36 & .83 \\
28 & 31.10 & 27.76 & .67 & .45 & .82 \\
\hline
\end{tabular}

Tabla 8

Escala de Manejo

\begin{tabular}{cccccc}
\hline Ítems & $\begin{array}{c}\text { Media de escala si } \\
\text { el elemento se ha } \\
\text { suprimido }\end{array}$ & $\begin{array}{c}\text { Varianza de escala } \\
\text { si el elemento se ha } \\
\text { suprimido }\end{array}$ & $\begin{array}{c}\text { Correlación total de } \\
\text { elementos corregida }\end{array}$ & $\begin{array}{c}\text { Correlación } \\
\text { múltiple al } \\
\text { cuadrado }\end{array}$ & $\begin{array}{c}\text { Alfa de Cronbach si } \\
\text { el elemento se ha } \\
\text { suprimido }\end{array}$ \\
\hline 2 & 11.21 & 15.26 & .41 & .19 & .67 \\
3 & 11.64 & 14.77 & .46 & .23 & .29 \\
4 & 11.25 & 14.54 & .48 & .36 & .65 \\
7 & 10.88 & 13.79 & .57 & .18 & .61 \\
11 & 10.64 & 15.03 & .38 & & .69 \\
\hline
\end{tabular}

Escala de Apego Ambivalente. Esta escala se conforma por los ítems 5, 6, 8 y 9. El Alfa de Cronbach de la escala fue $\alpha=.72$. En la tabla 9 se presentan la correlación entre los ítems de la escala y su análisis de consistencia interna.

\section{Validez convergente}

En la tabla 10 se presenta la correlación entre las diferentes escalas.

\section{Estructura Factorial de la escala SOC}

Se analizó el modelo factorial clásico de la escala SOC con sus tres dimensiones, en donde se encontró como resultados $\chi^{2}=1275.88, p=<.01, \mathrm{CFI}=.85$, RMSEA $=.07$ [.06-.07], SRMR=.04. En la figura 1 se muestra el modelo testeado con sus estimaciones estandarizadas.

Tabla 9

Escala de Apego Ambivalente

\begin{tabular}{cccccc}
\hline Ítems & $\begin{array}{c}\text { Media de escala si } \\
\text { el elemento se ha } \\
\text { suprimido }\end{array}$ & $\begin{array}{c}\text { Varianza de escala } \\
\text { si el elemento se ha } \\
\text { suprimido }\end{array}$ & $\begin{array}{c}\text { Correlación total de } \\
\text { elementos corregida }\end{array}$ & $\begin{array}{c}\text { Correlación } \\
\text { múltiple al } \\
\text { cuadrado }\end{array}$ & $\begin{array}{c}\text { Alfa de Cronbach si } \\
\text { el elemento se ha } \\
\text { suprimido }\end{array}$ \\
\hline 5 & 7.62 & 9.88 & .55 & .34 & .64 \\
6 & 8.22 & 9.98 & .46 & .23 & .69 \\
8 & 8.26 & 10.48 & .43 & .22 & .70 \\
9 & 8.37 & 8.83 & .60 & .39 & .60 \\
\hline
\end{tabular}

Tabla 10

Análisis de Correlación entre la SOC y Apego

\begin{tabular}{lcccc}
\hline \multicolumn{1}{c}{ Variables } & 1 & 2 & 3 & 4 \\
\hline 1. Comprensión SOC & 1 & & & \\
2. Manejo SOC & $.82^{* *}$ & 1 & & \\
3. Significado SOC & $.83^{* *}$ & $.87^{* *}$ & 1 & 1 \\
4. Apego evitativo & $-.30^{* *}$ & $-.24^{* *}$ & $-.27^{* *}$ & $.77^{* *}$ \\
5. Apego ambivalente & $-.30^{* *}$ & $-.28^{* *}$ & $-.30^{* *}$ & 1 \\
\hline
\end{tabular}

** La correlación es significativa en el nivel 0.01 (bilateral) 


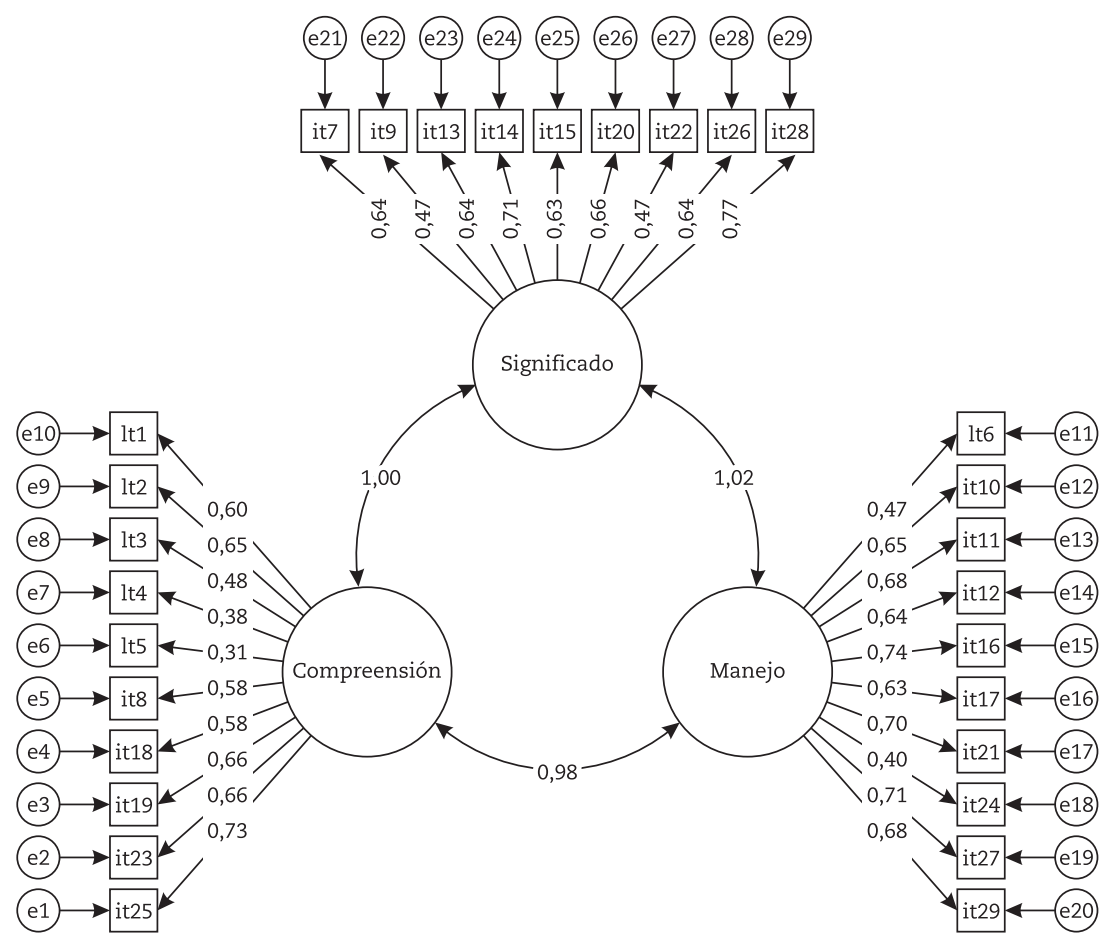

Figura 1. Modelo Factorial de la escala SOC

\section{Discusión y consideraciones finales}

En este artículo, se ha reportado una investigación que tuvo como finalidad evaluar las propiedades psicométricas de la escala SOC que permite valorar el sentido de coherencia, que en el caso particular, interesa para ser aplicada en población adulta de habla hispana, contextualizada en Ecuador.

La investigación planteó como hipótesis (a) que la escala SOC modificada y adaptada lingüísticamente al contexto ecuatoriano, presentará valores adecuados de confiabilidad, al ser valorada mediante el procedimiento de consistencia interna, (b) la escala SOC tendrá un adecuado valor de validez convergente al ser correlacionada con una escala que evalúe un constructo similar como lo el apego y (c) la escala SOC en su versión modificada y adaptada al contexto lingüístico de Ecuador presentará valores de bondad de ajuste adecuados en su estructura factorial de 3 dimensiones: significado, comprensión y manejo.

Los resultados obtenidos aportan evidencia en favor de la primera hipótesis planteada, ya que el valor de consistencia interna obtenido en las tres escalas de comprensión, manejo y significado se encuentran valores sobre .82, los cuales son interpretados como buenos valores al ser evaluados con el coeficiente de Alfa de Cronbach (Field, 2009), resultados que son concordantes con investigaciones previas en donde se ha encontrado parámetros de consistencia interna relacionados con los hallados en esta investigación (Eriksson \& Lindström, 2005; LizarbeChocarro et al., 2016).

En cuanto a la segunda hipótesis planteada, los resultados encontrados aportan evidencia en su favor, ya que las escalas SOC correlacionaron significativamente con la escala de apego en una magnitud entre mediana y grande, lo cual es un dato de validez a favor de la escala SOC para ser aplicada en el contexto ecuatoriano. Es importante mencionar que la escala de apego seguro fue eliminada del análisis de validez convergente, ya que sus valores de confiabilidad fueron muy bajos e interpretar una posible asociación con las variables evaluadas por la SOC, hubiera sido una posible correlación espuria y podríamos estar cayendo en el error tipo I de la investigación (Pérez-Tejada, 2008).

En relación a la tercera hipótesis, los resultados del análisis factorial confirmatorio, permiten identificar un cumplimiento aceptable del modelo teórico clásico de la SOC, lo cual, aporta en favor de la validez de constructo de la escala con las modificaciones lingüísticas realizadas que propician su aplicación en futuras investigaciones con población adulta de Ecuador. Es importante recalcar que el valor de $\mathrm{p}$ es significativo, no obstante, este valor es muy sensible a ser menor con tamaños de muestra como el de la presente investigación, por tal razón, al momento de interpretar el modelo propuesto tomamos como referencia los valores de CFI, RMSEA y SRMR que no son sensibles a este sesgo y son más significativos para interpretar la estructura misma del modelo testeado. 
Es importante recalcar, que uno de los cambios más relevantes que se llevó a cabo en la adaptación de la escala, es la modificación hecha en la construcción de las frases; pese a que algunas no se manipularon por parte del equipo de investigación y se mantuvieron en su versión original, otras fueron transformadas en el sentido de negativo a positivo, se simplificaron o se acortaron. Lo cual, sería el aporte principal de este estudio, ya que se propone una escala adaptada a la realidad lingüística de Ecuador, con propiedades psicométricas de validez y confiabilidad adecuadas para evaluar el sentido de coherencia.

Las modificaciones hechas en el sentido (de negativo a positivo) se realizaron bajo la premisa de que el cerebro procesa más fácilmente la información positiva que la negativa (Watzlawick, 2012). Además, al orientar la atención sobre los aspectos positivos más que sobre los negativos, se aumenta la concentración de energía y se incrementa el potencial de cada persona (Strano, 2002). De igual manera, se ha encontrado que el pensamiento negativo tiene correlación con el aumento de ansiedad, depresión, preocupación y disminución de la calidad de vida (Wadsworth et al., 2018), en cambio el pensamiento positivo puede mejorar la motivación y se sugiere además, que puede tener efectos positivos en el sistema atencional (Ishii et al., 2018).

La reducción de las frases, además promueve su fácil comprensión en lugar de tener frases largas que pueden prestarse a confusión. Un argumento en favor de cambio realizado tiene que ver con la atención que ejerce cierto grado de control sobre el funcionamiento cognitivo y previene la sobrecarga que puede aparecer por excesiva información, como es el caso de frases largas y complicadas (Morán, 2010).

En cuanto a los aportes de contar con la escala SOC validada en el contexto local, se puede resaltar el disponer de un instrumento validado para evaluar el sentido de coherencia, (b) su uso libre por parte de otros investigadores de Ecuador y otros contextos de habla hispana, (c) la posibilidad de que la línea de investigación sobre el sentido de coherencia siga creciendo en el contexto Latinoamericano, al tener un instrumento accesible para la realización de nuevos estudios y (d) la oportunidad que estudiantes de programas de pre y postgrado de Ecuador puedan aplicar este instrumento como operacionalización de la variable sentido de coherencia, dentro de sus trabajos de titulación. Por tanto, resulta necesario ejecutar esta investigación que tiene como finalidad validar la escala de sentido de coherencia - SOC 29 en el contexto ecuatoriano.

Gracias al planteamiento de la nueva escala modificada, es posible llevar a cabo investigaciones acerca del Sentido de Coherencia, usando este valioso instrumento en investigaciones descriptivas o correlacionales de un gran número de dificultades psicológicas. Esto se potencia debido a que la escala es de libre acceso, por lo que, las personas pueden acceder a ella y usarla en sus trabajos.
Como limitaciones se pueden mencionar el carácter subjetivo que siempre está implicado en la realización de una prueba de auto-reporte como la que se ha estudiado en esta investigación, que podría sesgar la información recolectada, ya que la persona al momento de responder los cuestionamientos realizados podría proteger su auto-concepto, generando respuestas sesgadas, no obstante, se controló esta condición de la prueba con una aplicación anónima y los resultados estadísticos encontrados, permiten interpretar como adecuadas las respuestas obtenidas.

Como segunda limitación, debemos declarar la localización geográfica de la muestra, que corresponde a una determinada ciudad de Latinoamérica, sin embargo, las características de Quito, lugar donde fue realizada la investigación, son similares a las de otras metrópolis del mundo, lo cual hace que esta propuesta científica pueda ser útil en otro contexto similar. Finalmente, una tercera limitación del estudio, tiene que ver con el tipo de muestreo aplicado, que fue no probabilístico, no obstante, esta limitación, así como las previas, motivan al equipo investigador a realizar nuevas investigaciones, donde podamos controlar estas limitaciones y generar más aportes empíricos en favor de la línea de investigación del sentido de coherencia en Latinoamérica.

Como investigación futura del trabajo realizado, se desprenden algunas líneas muy interesantes. Se puede estudiar más el apego y validar una escala adecuada al medio ecuatoriano. También se puede trabajar sobre el apego seguro y su relación con el sentido de coherencia puesto que, los resultados obtenidos señalan que existe una relación entre estas dos variables.

Finalmente, en cuanto a la escala del Sentido de Coherencia se puede ampliar la investigación mediante su propuesta como una escala breve, que permita su aplicación masiva y en menores tiempos. Además, es de interés analizar las propiedades psicométricas de la escala SOC mediante aplicaciones en población clínica psicológica como la depresión, suicidio, estrés, ansiedad, violencia, género y otras temáticas de interés para la psicología.

\section{Reconocimiento}

No hay menciones.

\section{Financimiento}

La presente investigación no recibió financimiento directo, fue costeada por los propios recursos de los autores.

\section{Contribuciones de los autores}

Todos los autores contribuyeron sustancialmente a la elaboración del diseño de la investigación, análisis e interpretación de los datos, así como a la revisión textual y aprobación de la versión final de este estudio. Todos los autores asumen la responsabilidad pública del contenido del manuscrito. 


\section{Disponibilidad de datos y materiales}

Todos los datos y sintaxis generados y analizados durante esta investigación serán tratados en completo secreto debido a los requisitos del Comité de Ética de Investigación en Humanos. Sin embargo, el conjunto de datos y sintaxis que respaldan las conclusiones de este artículo están disponibles previa solicitud razonable al autor principal del estudio.

\section{Conflicto de intereses}

Los autores declaran que no existen conflictos de intereses.

\section{Referencias}

Antonovsky, A. (1993). The structure and properties of the sense of coherence scale. Social Science and Medicine, 36(6), 725-733. doi: 10.1016/0277-9536(93)90033

Antonowsky, A. (1987). Unraveling the Mystery of Health: How People manage stress an stay well. San Francisco: Jossey-Bass.

Antonowsky, A. (1996). The salutogenic model as a theory to guide health promotion. Health Promotion International, 11(1), 11-18. doi: 10.1093/heapro/11.1.11

Besteiro, J., Álvarez, M., Lemos, S., Muñiz, J., Costas, C., \& Weruaga, A. (2008). Dimensiones de personalidad, sentido de coherencia y salud percibida en pacientes con un síndrome fibromiálgico. International Journal of Clinical and Health Psychology, 8(2), $411-427$.

Bowlby, J. (1998). El apego. Barcelona: Paidós.

Dejo, M. (2007). Sentido de coherencia, afrontamiento y sobrecarga en cuidadores familiares de ancianos con enfermedad crónica. Avances en Psicología Latinoamericana, 25(1) 64-71.

Dematteis, M., Grill, S., \& Posada, M. (2012). Sentido de coherencia y resiliencia: características salugénicas de personalidad. Buenos Aires: IV Congreso Internacional de Investigación y Práctica Profesional en Psicología. XIX Jornadas de Investigación y VIII Encuentro de Investigadores en Psicología del MERCOSUR.

Eriksson, M., \& Lindström, B. (2005). Validity of Antonovsky's sense of coherence scale: A systematic review. Journal of Epidemiology and Community Health, 59(6), 460-466. doi: 10.1136/jech.2003.018085

Field, A. (2009). Discovering Statistics Using SPSS. Third Edition. Los Angeles: SAGE.

Flensborg-Madsen, T., Ventegodt, S., \& Merrick, J. (2005). Why is Antonovsky's sense of coherence not correlated to physical health? Analysing Antonovsky's 29-item Sense of Coherence Scale (SOC-29). Scientific World Journal, 14(5), 767-776. doi: 10.1100/tsw.2005.89

Gómez, L. (17 de Octubre de 2017). Salutogénesis: ¿quées lo que mantiene al ser humano sano? Obtenido de Apyo emocional: http://apoyoemocional. univalle.edu.co/salutogenesis.pdf

Hernández, Z., Ehrenzweig, Y., \& Yépez, L. (2010). Sentido de coherencia y salud en personas adultas mayores autopercibidas como sanas. Revista Costarricense de Psicología, 29(43), 17-34. doi: 10.22544/rcps.v29i43.02

Ishii, A., Ishizuka, T., Muta, Y., Tanaka, M., Yamano, E., \& Watanabe, Y. (2018). The neural effects of positively and negatively re-experiencing mental fatigue sensation: a magnetoencephalography study. Experimental Brain Research, 236(6), 1735-1747. doi: 10.1007/s00221-0185260-0.

Lizarbe-Chocarro, M., Guillén-Grima, F., Aguinaga-Ontoso, I., \& Canga-Armayor, M. (2016). Validación del cuestionario Orientación a la Vida (OLQ-13) de Antonovsky en una muestra de estudiantes universitarios en Navarra. Anales del Sistema Sanitario de Navarra, 39(2) 237-248.

López-Martínez, C., Frías-Osuna, A., \& Del Pino-Casado, R. (2017). Sentido y coherencia y sobrecarga subjetiva, ansiedad y depresión en personas cuidadoreas de familiares mayores. Gaceta Sanitaria, 32(2), 1-6. doi: 10.1016/j.gaceta.2017.09.005

Malagón, M. (2016). El sentido de coherencia y el compromiso laboral de las enfermeras en el ámbito socio-sanitario de Girona. Girona: Universidad de Girona.

Mantas, S. (2017). Sentido de coherencia, Resiliencia y Salud Mental Positiva en los profesionales del Sistema de Emergencias Médicas. Girona: Universidad de Girona.

Martínez, E., Liébana, C., \& Morán, C. (2017). Relación entre el sentido de coherencia y el cansancio emocional en estudiantes universitarios. Psychology, Society \& Education, 9(3), 393-403. doi: 10.25115/psye.v9i3.861

Morán, E. (2010). Psicología general. Quito: Abya-Yala.

Ortiz-Granja, D. (2016). Escala de Apego. Quito-Ecuador: Pontificia Universidad Católica del Ecuador.

Paredes-Carbonell, J., Agulló-Cantos, J., Vera-Remartínez, E., \& Hernán-García, M. (2013). Sentido de coherencia y activos para la salud en jóvenes internos en centros de menores. Revista Española de Sanidad Penitenciaria, 15(3), 87-97.

Pérez-Tejada, H. (2008). Estadística para las ciencias sociales, del comportamiento y de la salud. México D.F.: CENGAGE Learning.

Rivera, F., López, A., Ramos, P., \& Moreno, C. (2011). Propiedades Psicométricas de la Escala Sentido de Coherencia (SOC-29) en adolescentes españoles. Journal of Child and Adolescent Psychology, 2(4), 11-39.

Salazar, J., Ruvalcaba, N., Figueroa, N., \& Pando, M. (2015). Sentido de coherencia y calidad de vida en trabajadores que participan en un programa de bienestar. Revista Iberoamericana de Psicología, Ciencia y Tecnología, 8(2), 91-98.

Saravia, J., Ibérico, C., \& Yearwood, K. (2014). Validation of Sense of Coherence (SOC) 13-ítem scale in a peruvian sample. Journal of Behavior. Health \& Social Issues, 6(2), 35-44. doi: 10.5460/jbhsi.v6.2.43847

Seligman, M. (1991). Aprenda optimismo. Santiago de Chile: Debolsillo.

Strano, A. (2002). El potencial del pensamiento positivo. España: Brahma Kumaris.

Ureña, P. (2008). Calidad de vida, sentido de coherencia y niveles de sedentarismo en académicos(as) y administrativos(as) del Campus Presbítero Benjamín Núñez. Movimiento Humano y Salud, 5(2), 1-15. doi: 10.15359/mhs.5-2.2

Ureña, P., \& Castro, C. (2009). Calidad de vida, sentido de coherencia y satisfacción laboral en profesores(as) de colegios técnicos en la Dirección Regional de Heredia. Revista Electrónica Educare, 13(1), 71-87. doi: 10.15359/ree.13-1.6

Velásquez, H., Cárdenas, V., Chávez, A., \& Montes de Oca, V. (2014). Comparación de dos formas de una escala de sentido de coherencia. Revista Intercontinental de Psicología y Educación, 16(2), 51-70. 
Virués, J., Martínez, P., Barrio, J., \& Lozano, L. (2007). Validación transcultural de la escala de Sentido de Coherencia de Antonovksy (OLQ13) en ancianos mayores de 70 años. Medicina Clínica, 128(13), 486-492. doi: 10.1157/13100935

Wadsworth, L., Forgeard, M., Hsu, K., Kertz, S., Treadway, M., \& Björgvinsson, T. (2018). Examining the Role or Repetitive Negative Thinking in Relations between Positiva and Negative Aspects of Self-compassión and Sympton Improvement during intensive treatment. Cognitive Therapy and Research, 42(3), 236-249. doi:10.1007/s10608-017-9887-0

Watzlawick, P. (2012). El lenguaje del cambio. Técnica de comunicación terapéutica. Barcelona: Herder.

\section{Sobre os autores}

Dorys Ortiz-Granja é psicóloga clínica, terapeuta familiar sistémica, docente titular de la Facultad de Psicología de la Pontificia Universidad Católica del Ecuador y lideresa de los procesos de formación continua.

Liliana Jayo-Suquillo é psicóloga Clínica, máster en estudios sociales con énfasis en género, docente titular de la Facultad de Psicología de la Pontificia Universidad Católica del Ecuador.

Carlos Ramos-Galarza é PhD en psicología clínica, docente titular de la Facultad de Psicología de la Pontificia Universidad Católica del Ecuador, investigador en proyectos de neurociencia y salud mental.

\section{Como citar este artigo}

Granja et al. (2020). Estructura Factorial del Sentido de Coherencia y su Relación de Apego. Avaliação Psicológica, 19(3), $298-309$. http://dx.doi.org/10.15689/ap.2020.1903.17132.08 